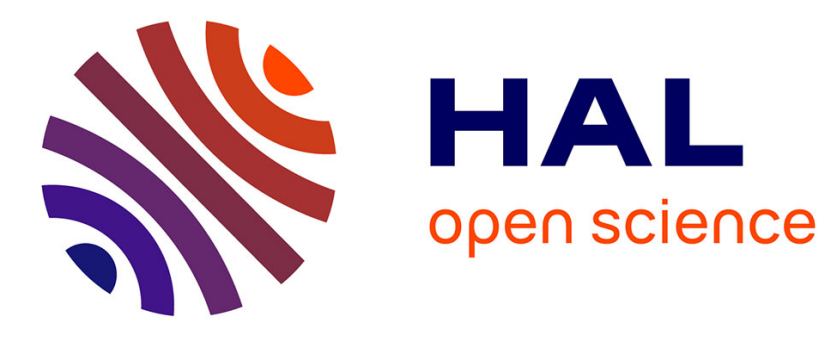

\title{
INARCH(1) processes: Higher-order moments and jumps
}

\author{
Christian H. Weiss
}

\section{To cite this version:}

Christian H. Weiss. INARCH(1) processes: Higher-order moments and jumps. Statistics and Probability Letters, 2010, 10.1016/j.spl.2010.08.001 . hal-00691785

\section{HAL Id: hal-00691785 \\ https://hal.science/hal-00691785}

Submitted on 27 Apr 2012

HAL is a multi-disciplinary open access archive for the deposit and dissemination of scientific research documents, whether they are published or not. The documents may come from teaching and research institutions in France or abroad, or from public or private research centers.
L'archive ouverte pluridisciplinaire HAL, est destinée au dépôt et à la diffusion de documents scientifiques de niveau recherche, publiés ou non, émanant des établissements d'enseignement et de recherche français ou étrangers, des laboratoires publics ou privés. 


\section{Accepted Manuscript}

INARCH(1) processes: Higher-order moments and jumps

Christian H. Weiß

PII:

S0167-7152(10)00224-5

DOI:

10.1016/j.spl.2010.08.001

Reference:

STAPRO 5760

To appear in: Statistics and Probability Letters

Received date: 26 May 2010

Revised date: 2 August 2010

Accepted date: 10 August 2010

Please cite this article as: Weiß, C.H., INARCH(1) processes: Higher-order moments and jumps. Statistics and Probability Letters (2010), doi:10.1016/j.spl.2010.08.001

This is a PDF file of an unedited manuscript that has been accepted for publication. As a service to our customers we are providing this early version of the manuscript. The manuscript will undergo copyediting, typesetting, and review of the resulting proof before it is published in its final form. Please note that during the production process errors may be discovered which could affect the content, and all legal disclaimers that apply to the journal pertain. 


\title{
INARCH(1) Processes: Higher-Order Moments and Jumps
}

\author{
Christian H. Weiß
}

\section{Full address:}

Christian H. Weiß

Darmstadt University of Technology

Department of Mathematics

Schlossgartenstraße 7

D-64289 Darmstadt

Germany

Email: weiss@mathematik.tu-darmstadt.de

Tel.: +49-6151-16-3787

Fax: +49-6151-16-6535 


\title{
INARCH(1) Processes: Higher-Order Moments and Jumps
}

\author{
Christian H. Weiß ${ }^{\mathrm{a}}$
}

${ }^{a}$ Department of Mathematics, Darmstadt University of Technology, Germany.

\begin{abstract}
The INARCH(1) model is a simple but practically relevant, two-parameter model for processes of overdispersed counts with an autoregressive serial dependence structure. We derive closed-form expressions for the joint (central) moments and cumulants of the INARCH(1) model up to order 4. These expressions are applied to derive moments of jumps in INARCH(1) processes. We illustrate this kind of application with a real-data example, and outline further potential applications.
\end{abstract}

Keywords: Count-data time series; cumulants; INARCH(1) model; jumps; moments; overdispersion.

\section{Introduction}

During the last years, a number of articles considered the analysis and modeling of count-data time series with overdispersion, i. e., with a variance greater than the mean, such that the marginal process distribution cannot be described by the popular Poisson model. Typical reasons for such overdispersion are the presence of positive correlation between the monitored events or a variation in the probability of the monitored events, see Weiß (2009a) and references therein, which are commonly observed in real-world applications. Some of these articles discuss ARMA-type models based on an appropriate type of thinning operation, see the survey by Weiß (2008a), but recently, another approach for modeling time series of overdispersed counts became quite popular: the INGARCH models, the integer-valued counterpart to the usual generalized autoregressive conditional heteroskedasticity models. The INGARCH models were introduced by Heinen (2003) and further investigated by Ferland et al. (2006); Fokianos et al. (2009); Fokianos \& Fried (2010); Weiß (2009a, 2010); Zhu \& Wang $(2009,2010)$. We consider the particular case of the INGARCH $(1,0)$ model in the following, and refer to it as the $\operatorname{INARCH(1)}$ model. This simple two-parameter model with its AR(1)-like serial dependence structure has already proved to be of great practical relevance, with concrete applications being reported for monthly claims counts (Weiß, 2009a), for download counts (Zhu \& Wang, 2010), and for monthly strike data (Weiß, 2010).

1.1 Definition (INARCH(1) Model) Let $\left(X_{t}\right)_{\mathbb{Z}}$ be a process with state space $\mathbb{N}_{0}=$ $\{0,1, \ldots\}$, let $\beta>0$ and $0<\alpha<1$. The process $\left(X_{t}\right)_{\mathbb{Z}}$ is said to follow an $\operatorname{INARCH}(1)$

Email address: weiss@mathematik.tu-darmstadt.de (Christian H. Weiß) 
model if $X_{t}$, conditioned on $X_{t-1}, X_{t-2}, \ldots$, is Poisson distributed according to Po( $\beta+$ $\left.\alpha \cdot X_{t-1}\right)$.

The INARCH(1) model of Definition 1.1 is a stationary, ergodic Markov chain (Ferland et al., 2006; Zhu \& Wang, 2009) with simple Poisson probabilities as the transition probabilities. All moments exist (Ferland et al., 2006), and its autocorrelation function $\rho(k):=\operatorname{Corr}\left[X_{t}, X_{t-k}\right]=\alpha^{k}$ like in the standard AR(1) case (Weiß, 2009a). The marginal distribution of the INARCH(1) model can be expressed in terms of its cumulants, which can be determined according to the recursive scheme provided by Weiß (2009a, 2010). In particular, using the abbreviation $f_{k}:=\beta / \prod_{i=1}^{k}\left(1-\alpha^{i}\right)$, the first four cumulants are given by

$$
\kappa_{1}=f_{1}, \quad \kappa_{2}=f_{2}, \quad \kappa_{3}=f_{3} \cdot\left(1+2 \alpha^{2}\right), \quad \kappa_{4}=f_{4} \cdot\left(1+6 \alpha^{2}+5 \alpha^{3}+6 \alpha^{5}\right),
$$

see Weiß (2009a). As a consequence of formula (1), mean $\mu$, variance $\sigma^{2}$, skewness and excess of $X_{t}$ are given by

$$
\frac{\beta}{1-\alpha}, \quad \frac{\beta}{(1-\alpha)\left(1-\alpha^{2}\right)}, \quad \frac{1+2 \alpha^{2}}{1+\alpha+\alpha^{2}} \cdot \sqrt{\frac{1+\alpha}{\beta}} \text { and } \frac{1+6 \alpha^{2}+5 \alpha^{3}+6 \alpha^{5}}{\beta\left(1+\alpha+\alpha^{2}\right)\left(1+\alpha^{2}\right)},
$$

respectively. Since $\sigma^{2}>\mu$, the INARCH(1) model allows to describe overdispersion.

In this article, we shall consider joint (central) moments and cumulants of the INARCH(1) model. In Section 2, we derive closed-form expressions for such moments and cumulants up to order 4. These expressions are applied in Section 3 to derive moments of jumps in INARCH(1) processes, and we illustrate this kind of application with a real-data example. Further potential applications are outlined in context of the conclusions in Section 4.

\section{Moments and Cumulants of INARCH(1) Processes}

The aim of this section is to provide closed-form expressions for the joint (central) moments and cumulants of the INARCH(1) model up to order 4. For this purpose, we introduce the following notations:

$$
\begin{aligned}
& \mu\left(s_{1}, \ldots, s_{r-1}\right):=E\left[X_{t} \cdot X_{t+s_{1}} \cdots X_{t+s_{r-1}}\right], \\
& \tilde{\mu}\left(s_{1}, \ldots, s_{r-1}\right):=E\left[\left(X_{t}-\mu\right)\left(X_{t+s_{1}}-\mu\right) \cdots\left(X_{t+s_{r-1}}-\mu\right)\right], \\
& \kappa\left(s_{1}, \ldots, s_{r-1}\right):=\operatorname{Cum}\left[X_{t}, X_{t+s_{1}}, \ldots, X_{t+s_{r-1}}\right],
\end{aligned}
$$

with $0 \leq s_{1} \leq \ldots \leq s_{r-1}$ and $r \in \mathbb{N}$. The case $r=1$ would correspond to the marginal mean $\mu:=E\left[X_{t}\right]=\beta /(1-\alpha)=f_{1}$.

2.1 Theorem (Moments of INARCH(1) Process) Let $\left(X_{t}\right)_{\mathbb{N}}$ be a stationary INARCH(1) process according to Definition 1.1.

(i) For any $k \geq 0$, we have $\mu(k)=f_{2} \cdot\left(\alpha^{k}+\beta(1+\alpha)\right)$.

(ii) For any $l \geq k \geq 0$, we have

$$
\mu(k, l)=\frac{\beta+1}{1-\alpha} \cdot f_{2} \cdot \alpha^{l}+f_{1} f_{2} \cdot \alpha^{l-k}-f_{3} \cdot(1-\alpha) \cdot \alpha^{l+k+1}+f_{1} \cdot \mu(k) .
$$


(iii) For any $m \geq l \geq k \geq 0$, we have

$$
\begin{aligned}
& \mu(k, l, m)=\alpha^{m-l} \cdot\left(\alpha^{2 l+k+2} \cdot f_{4} \cdot(1-\alpha)\left(2+2 \alpha-\alpha^{2}\right)\right. \\
& \quad-(\beta+2) \cdot f_{3} \cdot \alpha^{2 l+1}+\frac{f_{2} \cdot \alpha^{2 l-k}}{(1-\alpha)\left(1-\alpha^{2}\right)} \cdot(\alpha+2 \beta)-\alpha^{2(l-k)+1} \cdot f_{3} \cdot \beta \\
& \left.\quad+\frac{\beta+1}{1-\alpha} \cdot \mu(k, l)-f_{2} \cdot \mu(k) \cdot(\alpha+\beta(1+\alpha))\right)+f_{1} \cdot \mu(k, l) .
\end{aligned}
$$

The proof of Theorem 2.1 is provided in Appendix A.1. Now, we can apply Theorem 2.1 to derive analogous expressions for the joint central moments and cumulants.

2.2 Corollary (Central Moments and Cumulants) Let $\left(X_{t}\right)_{\mathbb{N}}$ be a stationary INARCH(1) process according to Definition 1.1.

(i) For any $k \geq 0$, we have $\tilde{\mu}(k)=\kappa(k)=f_{2} \cdot \alpha^{k}$.

(ii) For any $l \geq k \geq 0$, we have

$$
\tilde{\mu}(k, l)=\kappa(k, l)=f_{3} \cdot \alpha^{l} \cdot\left(1+\alpha+\alpha^{2}-(1-\alpha) \cdot \alpha^{k+1}\right) .
$$

(iii) For any $m \geq l \geq k \geq 0$, we have

$$
\begin{aligned}
& \kappa(k, l, m)=\alpha^{m} \cdot f_{4} \cdot\left((1-\alpha)\left(2+2 \alpha-\alpha^{2}\right) \cdot \alpha^{l+k+2}\right. \\
& \left.\quad-\left(1-\alpha^{4}\right) \cdot\left(2 \cdot \alpha^{l+1}+\alpha^{k+1}\right)+\left(1+\alpha+\alpha^{2}\right)\left(1+\alpha^{2}\right) \cdot\left(1+\alpha+\alpha^{l-k+1}\right)\right),
\end{aligned}
$$

and

$$
\tilde{\mu}(k, l, m)=\kappa(k, l, m)+f_{2}^{2} \cdot\left(\alpha^{m-l+k}+2 \alpha^{m+l-k}\right) .
$$

The proof of Corollary 2.2 is provided in Appendix A.2.

\section{Jumps in INARCH(1) Processes}

An interesting feature of a count-data process are jumps $J_{t}:=X_{t}-X_{t-1}$. Such jumps have already been investigated for the case of a Poisson INAR(1) process (Weiß, 2008b, 2009b) and a binomial AR(1) process (Weiß, 2009c). The extent of jumps in a count-data process reflects the extent of serial dependence: If the given count-data process exhibits an only small degree of serial dependence, then quite large jumps can be observed and vice versa. Therefore, the monitoring of jumps offers the potential of application to estimate model parameters related to the serial dependence structure, see, e. g., Weiß (2008b), or to construct control charts to detect changes in the serial dependence structure, as proposed by Weiß $(2009 \mathrm{~b}, \mathrm{c})$.

In view of these potential applications, we shall now investigate stochastic properties of jumps $J_{t}:=X_{t}-X_{t-1}$ in an INARCH(1) process. The distribution of $J_{t}$ might be described in terms of its cumulants. As shown in Appendix A.3, the following relation between the cumulant generating function (cgf) of $J_{t}$ and that of $X_{t}$ holds for an INARCH(1) process:

$$
\kappa_{J}(z)=\beta \cdot\left(e^{z}-1\right)+\kappa_{X}\left(\alpha \cdot\left(e^{z}-1\right)-z\right) .
$$




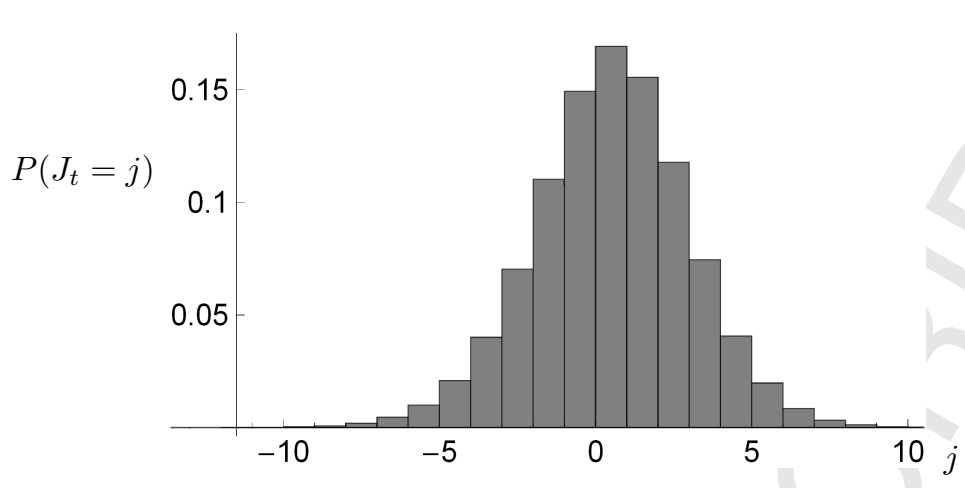

Figure 1: Distribution of $J_{t}$ for $\operatorname{INARCH}(1)$ process with $\beta=2$ and $\alpha=0.6$.

So taking derivatives on both sides of this equation, the cumulants of $J_{t}$ can be expressed in terms of the cumulants of $X_{t}$, also see formula (1). Alternatively, and we shall follow this approach in the following, one can apply the results of the previous Section 2 to derive expressions for moments of $J_{t}$ directly.

3.1 Proposition (Moments of Jumps in INARCH(1) Processes) Let $\left(X_{t}\right)_{\mathbb{N}}$ be a stationary INARCH(1) process according to Definition 1.1. Then

$$
\begin{aligned}
& E\left[J_{t}\right]=0, \quad E\left[J_{t}^{2}\right]=2 f_{2} \cdot(1-\alpha)=V\left[J_{t}\right], \quad E\left[J_{t}^{3}\right]=-3 f_{3} \alpha^{2}(1-\alpha)^{2}, \\
& E\left[J_{t}^{4}\right]=2 f_{4} \cdot(1-\alpha)^{2}\left(1+\alpha+4 \alpha^{2}+4 \alpha^{3}+2 \alpha^{4}+8 \alpha^{5}-2 \alpha^{6}\right)+12 f_{2}^{2} \cdot(1-\alpha)^{2} \\
& V\left[J_{t}^{2}\right]=2 f_{4} \cdot(1-\alpha)^{2}\left(1+\alpha+4 \alpha^{2}+4 \alpha^{3}+2 \alpha^{4}+8 \alpha^{5}-2 \alpha^{6}\right)+8 f_{2}^{2} \cdot(1-\alpha)^{2} .
\end{aligned}
$$

The proof is provided in Appendix A.4. It is quite interesting to note that in contrast to the case of the Poisson INAR(1) or binomial AR(1) process (Weiß, 2008b, 2009c), the distribution of jumps in an INARCH(1) process is not symmetric but negatively skewed. The effect of the dependence parameter $\alpha$ on the absolute extent of jumps becomes clear from $E\left[J_{t}^{2}\right]=2 \beta /\left(1-\alpha^{2}\right)$.

Concerning the autocorrelation structure of the process of jumps, we can simply adapt the result of Theorem 3 in Weiß (2009c), since it is determined only by the autocorrelation function of $\left(X_{t}\right)_{\mathbb{N}}$. So

$$
\rho_{J}(k)=-\frac{1-\alpha}{2} \cdot \alpha^{k-1}, \quad \rho_{\text {part }, J}(k)=-\frac{1-\alpha}{2+(k-1) \cdot(1-\alpha)} \quad \text { for } k \geq 1,
$$

which is always negative.

Let us conclude with a real-data example to see if and how the properties of Proposition 3.1 about theoretical moments can also be identified empirically in a given time series.

3.2 Example (Jumps in Strikes Data) We continue the real-data example from Section 5 of Weiß (2010), where a time series of monthly strike counts (January 1994 to December 2002) of total length $T=108$ was discussed and shown to be modeled very well by an INARCH(1) 
model with parameter values of about $\beta=2$ and $\alpha=0.6$. For such an INARCH(1) model, the jumps $J_{t}$ should have a $2^{\text {nd }}$ order moment (=variance) of $E\left[J_{t}^{2}\right]=6.25$, a $3^{\text {rd }}$ order moment of $E\left[J_{t}^{3}\right] \approx-1.72$ and thus a skewness of $\approx-0.11$. The moderate degree of negative skewness also becomes obvious from the estimated distribution shown in Figure 1, which was obtained from a simulated INARCH(1) time series of length $10^{6}$.

From the strikes data, we obtain a time series of 107 jumps. The empirical $2^{\text {nd }}$ order moment is computed as $\approx 6.47$, which is close to the theoretical value of 6.25 given above. For the empirical $3^{\text {rd }}$ order moment and skewness, we indeed observe negative values, but deviating more clearly from the theoretical ones given above: -8.64 and -0.53 , respectively. This, however, is no contradiction to the assumption of an underlying INARCH(1) process, but can be explained with the inherent variability of the estimators for these quantities: We simulated 10,000 time series of length 108 from an INARCH(1) process with parameters $\beta=2$ and $\alpha=0.6$. Each time, we computed the relevant empirical moments and used this data to analyze their stochastic properties. It turned out that

- the empirical $2^{\text {nd }}$ order moment has mean 6.28 , standard error 1.13 and skewness 0.63 such that the observed value of 6.47 is indeed very close to the expected one,

- the empirical $3^{\text {rd }}$ order moment has mean -1.82 , standard error 4.63 and skewness -0.80 such that the observed value of -8.64 deviates by less than 2 standard errors from the expected one,

- the skewness has mean -0.11 , standard error 0.26 and skewness -0.14 such that the observed value of -0.53 deviates by less than 2 standard errors from the expected one.

These results also show that the three estimators are at most moderately biased, but especially the $3^{\text {rd }}$ order statistics have quite large standard errors that do not allow to detect a significant deviation from 0 .

The last observation is somehow disappointing, since the property of asymmetric jumps distinguishes the INARCH(1) model, e. g., from the Poisson INAR(1) model and therefore offers the potential of deciding between both models. Such tools that allow to discriminate between different model families are generally of great practical importance, see, e. g., the discussion in Jung et al. (2006). Certainly, the standard errors decrease for an increasing length $T$ of the available time series, but only slowly (approximately with $1 / \sqrt{T}$ ) as shown by the following examples (for $T=250,500,1000,2500$ ): standard errors $2.99,2.10,1.49,0.93$ of empirical $3^{\text {rd }}$ order moments and standard errors $0.18,0.13,0.09,0.06$ of empirical skewness, respectively. These numbers are again obtained from simulating 10,000 time series each, and they show that quite long time series are necessary to be able to detect a significant asymmetry if empirical skewness or $3^{\text {rd }}$ order moments are used as statistics. Therefore, future research should try to find alternative statistics related to the symmetry behaviour of jumps, which might be applied already to short time series of counts.

\section{Conclusions and Future Research}

The INARCH(1) model is a simple but practically relevant, two-parameter model for processes of overdispersed counts with an AR(1)-like serial dependence structure. We derived closed-form expressions for its joint (central) moments and cumulants up to order 4. These expressions are particularly important for several types of application. As an example, we showed how to use them to derive moments of jumps in INARCH(1) 
processes. Such jumps offer themselves a lot of applications like parameter estimation or process monitoring, but in this context, see the discussed real-data example, it is also important to analyze moment properties of empirical quantities based on jumps. So an obvious issue for future research would be to apply the expressions for the joint moments presented in this article to derive (asymptotic) moments of jumps-based statistics. Jumps to the power of 3 might be useful for showing a significant asymmetry of the distribution of jumps (and therefore excluding, e. g., a Poisson INAR(1) model, see above), while squared jumps may be applied for parameter estimation: The squared difference estimator

$$
S D E:=\frac{1}{2(T-1)} \cdot \sum_{t=2}^{T}\left(X_{t}-X_{t-1}\right)^{2}=\frac{1}{2(T-1)} \cdot \sum_{t=2}^{T} J_{t}^{2}
$$

is an obviously unbiased estimator of the quantity $\sigma^{2} \cdot(1-\alpha)$ and hence a possible alternative to the first-order sample autocovariance as a (biased) estimator of $\sigma^{2} \cdot \alpha$.

Acknowledgements. The author would like to thank the referees for carefully reading the paper and for their comments which greatly improved the paper.

\section{References}

Bakouch, H.S., 2010. Higher-order moments, cumulants and spectral densities of the NGINAR(1) process. Stat. Meth. 7, 1-21.

Douglas, J.B., 1980. Analysis with standard contagious distributions. Int. Co-operative Publishing House, Fairland, Maryland USA.

Ferland, R., Latour, A., Oraichi, D., 2006. Integer-valued GARCH processes. Jour. Time Series Analysis 27(6), 923-942.

Fokianos, K., Rahbek, A., Tjøstheim, D., 2009. Poisson autoregression. Jour. Amer. Stat. Assoc. 104, 1430-1439.

Fokianos, K., Fried, T., 2010. Interventions in INGARCH processes. Jour. Time Series Analysis 31(3), $210-225$.

Heinen, A., 2003. Modelling Time Series Count Data: An Autoregressive Conditional Poisson Model. CORE Discussion Paper, 2003-63, University of Louvain, Belgium.

Jung, R.C., Kukuk, M., Liesenfeld, R., 2006. Time series of count data: modelling, estimation and diagnostics. Comp. Stat. Data Analysis 51, 2350-2364.

Weiß, C.H., 2008a. Thinning operations for modelling time series of counts - a survey. Adv. Stat. Analysis $92(3), 319-341$.

Weiß, C.H., 2008b. Serial dependence and regression of Poisson INARMA models. Jour. Stat. Planning Infer. 138(10), 2975-2990.

Weiß, C.H., 2009a. Modelling time series of counts with overdispersion. Stat. Meth. Appl. 18(4), 507-519.

Weiß, C.H., 2009b. Controlling jumps in correlated processes of Poisson counts. Appl. Stoch. Models Busin. Industry 25(5), 551-564.

Weiß, C.H., 2009c. Jumps in binomial AR(1) processes. Stat. Prob. Letters 79(19), 2012-2019.

Weiß, C.H., 2010. The INARCH(1) model for overdispersed time series of counts. Comm. Stat. - Simul. Comp 39(6), 1269-1291.

Zhu, F., Wang, D., 2009. Estimation and testing for a Poisson autoregressive model. To appear in Metrika.

Zhu, F., Wang, D., 2010. Diagnostic checking integer-valued $\operatorname{ARCH}(p)$ models using conditional residual autocorrelations. Comp. Stat. Data Analysis 54, 496-508.

\section{Appendix A. Proofs}

Appendix A.1. Proof of Theorem 2.1

Let $\left(X_{t}\right)_{\mathbb{N}}$ be a stationary INARCH(1) process according to Definition 1.1. Using the notations of formula (3), an explicit expression for the case $r=2$ is easily derived using that $V\left[X_{t}\right]=f_{2}$ 
according to formula (1) and that the autocorrelation function equals $\rho(k)=\alpha^{k}$ :

$$
\mu(k)=\operatorname{Cov}\left[X_{t}, X_{t+k}\right]+E\left[X_{t}\right]^{2}=f_{2} \cdot \alpha^{k}+f_{1}^{2}=f_{2} \cdot\left(\alpha^{k}+\beta(1+\alpha)\right) .
$$

Before we continue with higher-order moments, we need the following conditional moments:

$$
\begin{aligned}
E\left[X_{t} \mid X_{t-1}, \ldots\right]= & \alpha \cdot X_{t-1}+\beta, \\
E\left[X_{t}^{2} \mid X_{t-1}, \ldots\right]= & \alpha^{2} \cdot X_{t-1}^{2}+\alpha(2 \beta+1) \cdot X_{t-1}+\beta(\beta+1), \\
E\left[X_{t}^{3} \mid X_{t-1}, \ldots\right]= & \alpha^{3} \cdot X_{t-1}^{3}+3 \alpha^{2}(\beta+1) \cdot X_{t-1}^{2} \\
& +\alpha\left(3 \beta^{2}+6 \beta+1\right) \cdot X_{t-1}+\beta\left(\beta^{2}+3 \beta+1\right) .
\end{aligned}
$$

(Appendix A.1)

These expressions follow from the first three moments of the Poisson distribution, since the conditional distribution of $X_{t}$ given $X_{t-1}, \ldots$ is $\operatorname{Po}\left(\beta+\alpha \cdot X_{t-1}\right)$.

\section{Third-Order Moments}

In the following, we shall use the well-known formula $E[X]=E[E[X \mid Z]]$ for conditional expectations together with formula (Appendix A.1). To derive an explicit expression for $\mu(k, l)$ with $0 \leq k \leq l$, we distinguish between the following three cases:

Case 1: $l>k$. Then we have

$$
\begin{aligned}
\mu(k, l) & =E\left[X_{t} X_{t+k} \cdot E\left[X_{t+l} \mid X_{t+l-1}, \ldots\right]\right]=\alpha \cdot \mu(k, l-1)+\beta \cdot \mu(k) \\
& =\cdots=\alpha^{l-k} \cdot \mu(k, k)+\beta \cdot \mu(k) \cdot \sum_{j=0}^{l-k-1} \alpha^{j} \\
& =\alpha^{l-k} \cdot\left(\mu(k, k)-f_{1} \cdot \mu(k)\right)+f_{1} \cdot \mu(k) .
\end{aligned}
$$

Case 2: $l=k>0$. Then we have

$$
\begin{aligned}
& \mu(k, k)=E\left[X_{t} \cdot E\left[X_{t+k}^{2} \mid X_{t+k-1}, \ldots\right]\right] \\
& =\alpha^{2} \cdot \mu(k-1, k-1)+\alpha(2 \beta+1) \cdot \mu(k-1)+\beta(\beta+1) \cdot \mu \\
& =\alpha^{2} \cdot \mu(k-1, k-1)+(2 \beta+1) \cdot f_{2} \cdot \alpha^{k}+\alpha(2 \beta+1) \cdot f_{1}^{2}+(\beta+1) \cdot(1-\alpha) \cdot f_{1}^{2} \\
& =\alpha^{2} \cdot \mu(k-1, k-1)+(2 \beta+1) \cdot f_{2} \cdot \alpha^{k}+\left(1-\alpha^{2}\right) \cdot f_{1} \cdot \mu(0) \\
& =\ldots=\alpha^{2 k} \cdot \mu(0,0)+(2 \beta+1) \cdot f_{2} \cdot \sum_{j=0}^{k-1} \alpha^{k+j}+\left(1-\alpha^{2}\right) \cdot f_{1} \cdot \mu(0) \cdot \sum_{j=0}^{k-1} \alpha^{2 j} \\
& =\alpha^{2 k} \cdot\left(\mu(0,0)-\frac{2 \beta+1}{1-\alpha} \cdot f_{2}-f_{1} \cdot \mu(0)\right)+\frac{2 \beta+1}{1-\alpha} \cdot f_{2} \cdot \alpha^{k}+f_{1} \cdot \mu(0) .
\end{aligned}
$$

Case 3: $l=k=0$. Then we have according to formula [1,5] in Douglas (1980) and formula (1) that

$$
\begin{aligned}
& \mu(0,0)=E\left[X_{t}^{3}\right]=\kappa_{3}+3 \cdot \kappa_{2} \cdot \mu+\mu^{3}=f_{3} \cdot\left(1+2 \alpha^{2}\right)+3 \cdot f_{2} \cdot f_{1}+f_{1}^{3} \\
& =\left(\frac{f_{2}}{1-\alpha}-f_{3} \cdot \alpha(1-\alpha)\right)+f_{2} \cdot \frac{3 \beta}{1-\alpha}+f_{2} \cdot \frac{\beta^{2}(1+\alpha)}{1-\alpha} \\
& =\frac{f_{2}}{1-\alpha} \cdot(1+2 \beta)+\frac{f_{2}}{1-\alpha} \cdot \beta(1+\beta(1+\alpha))-f_{3} \cdot \alpha(1-\alpha)=\frac{1+2 \beta}{1-\alpha} \cdot f_{2}+f_{1} \cdot \mu(0)-f_{3} \cdot \alpha(1-\alpha) .
\end{aligned}
$$

So the above formula for $\mu(k, k)$ simplifies to

$$
\mu(k, k)=-f_{3} \cdot \alpha(1-\alpha) \cdot \alpha^{2 k}+\frac{2 \beta+1}{1-\alpha} \cdot f_{2} \cdot \alpha^{k}+f_{1} \cdot \mu(0),
$$

which also holds for $k=0$, and it follows that

$$
\begin{aligned}
& \mu(k, l)=\alpha^{l-k} \cdot\left(\mu(k, k)-f_{1} \cdot \mu(k)\right)+f_{1} \cdot \mu(k) \\
& =\alpha^{l-k} \cdot\left(-f_{3} \cdot \alpha(1-\alpha) \cdot \alpha^{2 k}+\left(\frac{2 \beta+1}{1-\alpha}-f_{1}\right) \cdot f_{2} \cdot \alpha^{k}+f_{1} \cdot\left(\mu(0)-f_{2} \cdot \beta(1+\alpha)\right)\right)+f_{1} \cdot \mu(k) \\
& =\alpha^{l-k} \cdot\left(-f_{3} \cdot \alpha(1-\alpha) \cdot \alpha^{2 k}+\frac{\beta+1}{1-\alpha} \cdot f_{2} \cdot \alpha^{k}+f_{1} \cdot f_{2}\right)+f_{1} \cdot \mu(k)
\end{aligned}
$$

holds for any $0 \leq k \leq l$. 


\section{Fourth-Order Moments}

To derive an explicit expression for $\mu(k, l, m)$ with $0 \leq k \leq l \leq m$, we proceed in a similar way as above and distinguish between the following four cases:

Case 1: $m>l$. Then we have as above that

$$
\mu(k, l, m)=\ldots=\alpha^{m-l} \cdot\left(\mu(k, l, l)-f_{1} \cdot \mu(k, l)\right)+f_{1} \cdot \mu(k, l) .
$$

Case 2: $m=l>k$. Then we have

$$
\begin{aligned}
\mu & (k, l, l)=E\left[X_{t} X_{t+k} \cdot E\left[X_{t+l}^{2} \mid X_{t+l-1}, \ldots\right]\right] \\
= & \alpha^{2} \cdot \mu(k, l-1, l-1)+\alpha(2 \beta+1) \cdot \mu(k, l-1)+\beta(\beta+1) \cdot \mu(k) \\
= & \alpha^{2} \cdot \mu(k, l-1, l-1)+\beta(\beta+1) \cdot \mu(k)+\alpha(2 \beta+1) \cdot f_{1} \cdot \mu(k) \\
& +\alpha^{l} \cdot(2 \beta+1) \cdot\left(-f_{3} \cdot \alpha(1-\alpha) \cdot \alpha^{k}+\frac{\beta+1}{1-\alpha} \cdot f_{2}+f_{1} \cdot f_{2} \cdot \alpha^{-k}\right) \\
= & \alpha^{2} \cdot \mu(k, l-1, l-1)+f_{1} \cdot(1+\beta(1+\alpha)) \cdot \mu(k) \\
& +\alpha^{l} \cdot(2 \beta+1) \cdot\left(-f_{3} \cdot \alpha(1-\alpha) \cdot \alpha^{k}+\frac{\beta+1}{1-\alpha} \cdot f_{2}+f_{1} \cdot f_{2} \cdot \alpha^{-k}\right) \\
= & \ldots=\alpha^{2(l-k)} \cdot \mu(k, k, k)+f_{1} \cdot(1+\beta(1+\alpha)) \cdot \mu(k) \cdot \sum_{j=0}^{l-k-1} \alpha^{2 j} \\
& +(2 \beta+1) \cdot\left(-f_{3} \cdot \alpha(1-\alpha) \cdot \alpha^{k}+\frac{\beta+1}{1-\alpha} \cdot f_{2}+f_{1} \cdot f_{2} \cdot \alpha^{-k}\right) \cdot \sum_{j=0}^{l-k-1} \alpha^{l+j} \\
= & \alpha^{2(l-k)} \cdot \mu(k, k, k)+f_{2} \cdot(1+\beta(1+\alpha)) \cdot \mu(k) \cdot\left(1-\alpha^{2(l-k)}\right) \\
& +\frac{2 \beta+1}{1-\alpha} \cdot\left(-f_{3} \cdot \alpha(1-\alpha) \cdot \alpha^{k}+\frac{\beta+1}{1-\alpha} \cdot f_{2}+f_{1} \cdot f_{2} \cdot \alpha^{-k}\right) \cdot\left(\alpha^{l}-\alpha^{2 l-k}\right) \\
= & \alpha^{2(l-k)} \cdot \mu(k, k, k)-f_{2}^{2} \cdot(1+\beta(1+\alpha)) \cdot \alpha^{2 l-k}-\frac{2 \beta+1}{1-\alpha} \cdot \frac{\beta+1}{1-\alpha} \cdot f_{2} \cdot \alpha^{2 l-k} \\
& -f_{2}^{2} \cdot(1+\beta(1+\alpha)) \cdot \beta(1+\alpha) \cdot \alpha^{2(l-k)}-\frac{2 \beta+1}{1-\alpha} \cdot f_{1} \cdot f_{2} \cdot \alpha^{2(l-k)} \\
& +\frac{2 \beta+1}{1-\alpha} \cdot f_{3} \cdot \alpha(1-\alpha) \cdot \alpha^{2 l}+f_{2} \cdot(1+\beta(1+\alpha)) \cdot \mu(k) \\
& +\frac{2 \beta+1}{1-\alpha} \cdot\left(-f_{3} \cdot \alpha(1-\alpha) \cdot \alpha^{k}+\frac{\beta+1}{1-\alpha} \cdot f_{2}+f_{1} \cdot f_{2} \cdot \alpha^{-k}\right) \cdot \alpha^{l} \\
= & \alpha^{2(l-k)} \cdot \mu(k, k, k)-\frac{f_{2} \cdot \alpha^{2 l-k}}{(1-\alpha)\left(1-\alpha^{2}\right)} \cdot\left(\beta+\beta^{2}(1+\alpha)+(1+\alpha)(2 \beta+1)(\beta+1)\right) \\
& -f_{2}^{2} \cdot(1+\alpha) \cdot \alpha^{2(l-k)} \cdot\left(\beta+\beta^{2}(1+\alpha)+2 \beta+1\right) \\
& +(2 \beta+1) \cdot f_{3} \cdot \alpha^{2 l+1}+\mu(0) \cdot \mu(k)+\frac{2 \beta+1}{1-\alpha} \cdot\left(\mu(k, l)-f_{1} \cdot \mu(k)\right) \\
= & \alpha^{2(l-k)} \cdot \mu(k, k, k)-\frac{f_{2} \cdot \alpha^{2 l-k}}{(1-\alpha)\left(1-\alpha^{2}\right)} \cdot\left(1+\alpha+(4+3 \alpha) \beta+3(1+\alpha) \beta^{2}\right)+(2 \beta+1) \cdot f_{3} \cdot \alpha^{2 l+1} \\
& -\alpha^{2(l-k)} \cdot\left(f_{1} \cdot \mu(0,0)+f_{3} \cdot \alpha \beta\right)+\frac{2 \beta+1}{1-\alpha} \cdot \mu(k, l)-f_{2} \cdot \mu(k) \cdot(\alpha+\beta(1+\alpha)) \\
&
\end{aligned}
$$


Case 3: $m=l=k>0$. Then we have

$$
\begin{aligned}
& \mu(k, k, k)=E\left[X_{t} \cdot E\left[X_{t+k}^{3} \mid X_{t+k-1}, \ldots\right]\right] \\
& =\alpha^{3} \cdot \mu(k-1, k-1, k-1)+3 \alpha^{2}(\beta+1) \cdot \mu(k-1, k-1) \\
& +\alpha\left(3 \beta^{2}+6 \beta+1\right) \cdot \mu(k-1)+\beta\left(\beta^{2}+3 \beta+1\right) \cdot \mu \\
& =\alpha^{3} \cdot \mu(k-1, k-1, k-1)-3(\beta+1) \cdot f_{3} \cdot \alpha(1-\alpha) \cdot \alpha^{2 k} \\
& +3 \alpha(\beta+1) \cdot \frac{2 \beta+1}{1-\alpha} \cdot f_{2} \cdot \alpha^{k}+\left(3 \beta^{2}+6 \beta+1\right) \cdot f_{2} \cdot \alpha^{k} \\
& +3 \alpha^{2}(\beta+1) \cdot f_{1} \cdot \mu(0)+\alpha\left(3 \beta^{2}+6 \beta+1\right) \cdot f_{2} \cdot \beta(1+\alpha)+\beta\left(\beta^{2}+3 \beta+1\right) \cdot f_{1} \\
& =\alpha^{3} \cdot \mu(k-1, k-1, k-1)-3(\beta+1) \cdot f_{3} \cdot \alpha(1-\alpha) \cdot \alpha^{2 k} \\
& +\frac{f_{2}}{1-\alpha} \cdot\left(1+2 \alpha+3(2+\alpha) \beta+3(1+\alpha) \beta^{2}\right) \cdot \alpha^{k} \\
& +f_{1} f_{2} \cdot\left(1+2 \alpha^{2}+3\left(1+\alpha+\alpha^{2}\right) \beta+(1+\alpha)\left(1+\alpha+\alpha^{2}\right) \beta^{2}\right) \\
& =\alpha^{3} \cdot \mu(k-1, k-1, k-1)-3(\beta+1) \cdot f_{3} \cdot \alpha(1-\alpha) \cdot \alpha^{2 k} \\
& +\frac{f_{2}}{1-\alpha} \cdot\left(1+2 \alpha+3(2+\alpha) \beta+3(1+\alpha) \beta^{2}\right) \cdot \alpha^{k}+f_{1} \cdot\left(1-\alpha^{3}\right) \cdot \mu(0,0) \\
& =\ldots=\alpha^{3 k} \cdot \mu(0,0,0)-3(\beta+1) \cdot f_{3} \cdot \alpha(1-\alpha) \cdot \sum_{j=0}^{k-1} \alpha^{2 k+j} \\
& +\frac{f_{2}}{1-\alpha} \cdot\left(1+2 \alpha+3(2+\alpha) \beta+3(1+\alpha) \beta^{2}\right) \cdot \sum_{j=0}^{k-1} \alpha^{k+2 j}+f_{1} \cdot\left(1-\alpha^{3}\right) \cdot \mu(0,0) \cdot \sum_{j=0}^{k-1} \alpha^{3 j} \\
& =\alpha^{3 k} \cdot \mu(0,0,0)-3(\beta+1) \cdot f_{3} \cdot \alpha \cdot\left(\alpha^{2 k}-\alpha^{3 k}\right) \\
& +\frac{f_{2}}{(1-\alpha)\left(1-\alpha^{2}\right)} \cdot\left(1+2 \alpha+3(2+\alpha) \beta+3(1+\alpha) \beta^{2}\right) \cdot\left(\alpha^{k}-\alpha^{3 k}\right)+f_{1} \cdot \mu(0,0) \cdot\left(1-\alpha^{3 k}\right) \\
& =\alpha^{3 k} \cdot \mu(0,0,0)+3(\beta+1) \cdot f_{3} \cdot \alpha \cdot \alpha^{3 k} \\
& -\frac{f_{2}}{(1-\alpha)\left(1-\alpha^{2}\right)} \cdot\left(1+2 \alpha+3(2+\alpha) \beta+3(1+\alpha) \beta^{2}\right) \cdot \alpha^{3 k}-f_{1} \cdot \mu(0,0) \cdot \alpha^{3 k} \\
& +\frac{f_{2} \cdot \alpha^{k}}{(1-\alpha)\left(1-\alpha^{2}\right)} \cdot\left(1+2 \alpha+3(2+\alpha) \beta+3(1+\alpha) \beta^{2}\right)+f_{1} \cdot \mu(0,0)-3(\beta+1) \cdot f_{3} \cdot \alpha^{2 k+1} \\
& =\alpha^{3 k} \cdot\left(\mu(0,0,0)-\frac{f_{3}}{1-\alpha^{2}} \cdot\left(1+3 \alpha^{2}+5 \alpha^{3}+\left(7+7 \alpha+11 \alpha^{2}+8 \alpha^{3}\right) \beta\right.\right. \\
& \left.\left.+6(1+\alpha)\left(1+\alpha+\alpha^{2}\right) \beta^{2}+(1+\alpha)^{2}\left(1+\alpha+\alpha^{2}\right) \beta^{3}\right)\right) \\
& +\frac{f_{2} \cdot \alpha^{k}}{(1-\alpha)\left(1-\alpha^{2}\right)} \cdot\left(1+2 \alpha+3(2+\alpha) \beta+3(1+\alpha) \beta^{2}\right)+f_{1} \cdot \mu(0,0)-3(\beta+1) \cdot f_{3} \cdot \alpha^{2 k+1} \\
& =\alpha^{3 k} \cdot\left(\mu(0,0,0)-f_{4} \cdot\left(1+4 \alpha^{2}+5 \alpha^{3}+3 \alpha^{4}+5 \alpha^{5}+\left(1+\alpha^{2}\right)\left(7+7 \alpha+11 \alpha^{2}+8 \alpha^{3}\right) \beta\right.\right. \\
& \left.\left.+6(1+\alpha)\left(1+\alpha^{2}\right)\left(1+\alpha+\alpha^{2}\right) \beta^{2}+(1+\alpha)^{2}\left(1+\alpha^{2}\right)\left(1+\alpha+\alpha^{2}\right) \beta^{3}\right)\right) \\
& +\frac{f_{2} \cdot \alpha^{k}}{(1-\alpha)\left(1-\alpha^{2}\right)} \cdot\left(1+2 \alpha+3(2+\alpha) \beta+3(1+\alpha) \beta^{2}\right)+f_{1} \cdot \mu(0,0)-3(\beta+1) \cdot f_{3} \cdot \alpha^{2 k+1} \text {. }
\end{aligned}
$$

Case 4: $m=l=k=0$. Then we have according to formula [1,5] in Douglas (1980) and formula (1) that

$$
\begin{aligned}
& \mu(0,0,0)=E\left[X_{t}^{4}\right]=\kappa_{4}+4 \cdot \kappa_{3} \cdot \mu+3 \kappa_{2}^{2}+6 \cdot \kappa_{2} \cdot \mu^{2}+\mu^{4} \\
& =f_{4} \cdot\left(1+6 \alpha^{2}+5 \alpha^{3}+6 \alpha^{5}+\left(1+\alpha^{2}\right)\left(7+7 \alpha+11 \alpha^{2}+8 \alpha^{3}\right) \beta\right. \\
& \left.\quad+6(1+\alpha)\left(1+\alpha^{2}\right)\left(1+\alpha+\alpha^{2}\right) \beta^{2}+(1+\alpha)^{2}\left(1+\alpha^{2}\right)\left(1+\alpha+\alpha^{2}\right) \beta^{3}\right) .
\end{aligned}
$$

So the above formula for $\mu(k, k, k)$ simplifies to

$$
\begin{gathered}
\mu(k, k, k)=\alpha^{3 k} \cdot f_{4} \cdot \alpha^{2}(1-\alpha)\left(2+2 \alpha-\alpha^{2}\right)-3(\beta+1) \cdot f_{3} \cdot \alpha^{2 k+1} \\
+\frac{f_{2} \cdot \alpha^{k}}{(1-\alpha)\left(1-\alpha^{2}\right)} \cdot\left(1+2 \alpha+3(2+\alpha) \beta+3(1+\alpha) \beta^{2}\right)+f_{1} \cdot \mu(0,0) .
\end{gathered}
$$


Inserting into the formula for $\mu(k, l, l)$, we obtain

$$
\begin{aligned}
& \mu(k, l, l)=\alpha^{2(l-k)} \cdot \mu(k, k, k)-\frac{f_{2} \cdot \alpha^{2 l-k}}{(1-\alpha)\left(1-\alpha^{2}\right)} \cdot\left(1+\alpha+(4+3 \alpha) \beta+3(1+\alpha) \beta^{2}\right) \\
& \quad-\alpha^{2(l-k)} \cdot\left(f_{1} \cdot \mu(0,0)+f_{3} \cdot \alpha \beta\right)+(2 \beta+1) \cdot f_{3} \cdot \alpha^{2 l+1} \\
& \quad+\frac{2 \beta+1}{1-\alpha} \cdot \mu(k, l)-f_{2} \cdot \mu(k) \cdot(\alpha+\beta(1+\alpha)) \\
& =\alpha^{2 l+k} \cdot f_{4} \cdot \alpha^{2}(1-\alpha)\left(2+2 \alpha-\alpha^{2}\right)-3(\beta+1) \cdot f_{3} \cdot \alpha^{2 l+1}+(2 \beta+1) \cdot f_{3} \cdot \alpha^{2 l+1} \\
& \quad+\frac{f_{2} \cdot \alpha^{2 l-k}}{(1-\alpha)\left(1-\alpha^{2}\right)} \cdot\left(\left(1+2 \alpha+3(2+\alpha) \beta+3(1+\alpha) \beta^{2}\right)-\left(1+\alpha+(4+3 \alpha) \beta+3(1+\alpha) \beta^{2}\right)\right) \\
& \quad+\alpha^{2(l-k)} \cdot f_{1} \cdot \mu(0,0)-\alpha^{2(l-k)} \cdot\left(f_{1} \cdot \mu(0,0)+f_{3} \cdot \alpha \beta\right) \\
& \quad+\frac{2 \beta+1}{1-\alpha} \cdot \mu(k, l)-f_{2} \cdot \mu(k) \cdot(\alpha+\beta(1+\alpha)) \\
& =\alpha^{2 l+k} \cdot f_{4} \cdot \alpha^{2}(1-\alpha)\left(2+2 \alpha-\alpha^{2}\right)-(\beta+2) \cdot f_{3} \cdot \alpha^{2 l+1}+\frac{f_{2} \cdot \alpha^{2 l-k}}{(1-\alpha)\left(1-\alpha^{2}\right)} \cdot(\alpha+2 \beta) \\
& \quad-\alpha^{2(l-k)} \cdot f_{3} \cdot \alpha \beta+\frac{2 \beta+1}{1-\alpha} \cdot \mu(k, l)-f_{2} \cdot \mu(k) \cdot(\alpha+\beta(1+\alpha)) .
\end{aligned}
$$

So it follows that for all $0 \leq k \leq l \leq m$, we have

$$
\begin{aligned}
& \mu(k, l, m)=\alpha^{m-l} \cdot\left(\mu(k, l, l)-f_{1} \cdot \mu(k, l)\right)+f_{1} \cdot \mu(k, l) \\
& =\alpha^{m-l} \cdot\left(\alpha^{2 l+k} \cdot f_{4} \cdot \alpha^{2}(1-\alpha)\left(2+2 \alpha-\alpha^{2}\right)-(\beta+2) \cdot f_{3} \cdot \alpha^{2 l+1}+\frac{f_{2} \cdot \alpha^{2 l-k}}{(1-\alpha)\left(1-\alpha^{2}\right)} \cdot(\alpha+2 \beta)\right. \\
& \left.-\alpha^{2(l-k)} \cdot f_{3} \cdot \alpha \beta+\frac{\beta+1}{1-\alpha} \cdot \mu(k, l)-f_{2} \cdot \mu(k) \cdot(\alpha+\beta(1+\alpha))\right)+f_{1} \cdot \mu(k, l) .
\end{aligned}
$$

\section{Appendix A.2. Proof of Corollary 2.2}

In the following, we shall apply the general relations between joint moments and joint cumulants stated by Bakouch (2010), as well as the notations of formula (3).

\section{Second-Order Central Moments and Cumulants}

For $k \geq 0$, we have $\kappa(k)=\mu(k)-\mu^{2}=\operatorname{Cov}\left[X_{t}, X_{t+k}\right]=f_{2} \cdot \alpha^{k}$, and obviously, also $\tilde{\mu}(k)=\operatorname{Cov}\left[X_{t}, X_{t+k}\right]$ coincides with this expression.

\section{Third-Order Central Moments and Cumulants}

For $l \geq k \geq 0$, we have

$$
\begin{aligned}
\kappa(k, l) & =\mu(k, l)-\mu \cdot \mu(k)-\mu \cdot\left(\mu(l-k)+\mu(l)-2 \mu^{2}\right) \\
& =\frac{\beta+1}{1-\alpha} \cdot f_{2} \cdot \alpha^{l}+f_{1} f_{2} \cdot \alpha^{l-k}-f_{3} \cdot(1-\alpha) \cdot \alpha^{l+k+1}-f_{1} \cdot\left(f_{2} \cdot \alpha^{l-k}+f_{2} \cdot \alpha^{l}\right) \\
& =\frac{f_{2}}{1-\alpha} \cdot \alpha^{l}-f_{3} \cdot(1-\alpha) \cdot \alpha^{l+k+1}=f_{3} \cdot \alpha^{l} \cdot\left(1+\alpha+\alpha^{2}-(1-\alpha) \cdot \alpha^{k+1}\right) .
\end{aligned}
$$

Furthermore, it follows that simply

$$
\begin{aligned}
\tilde{\mu}(k, l) & =E\left[\left(X_{t}-\mu\right)\left(X_{t+k}-\mu\right)\left(X_{t+l}-\mu\right)\right] \\
& =E\left[\left(X_{t} X_{t+k}-\mu \cdot X_{t}-\mu \cdot X_{t+k}+\mu^{2}\right) \cdot X_{t+l}\right]-\mu \cdot \tilde{\mu}(k) \\
& =\mu(k, l)-\mu \cdot \mu(l)-\mu \cdot \mu(l-k)+\mu^{3}-\mu \cdot \mu(k)+\mu^{3}=\kappa(k, l) .
\end{aligned}
$$


Fourth-Order Central Moments and Cumulants For $m \geq l \geq k \geq 0$, we have

$$
\begin{aligned}
\kappa & (k, l, m)=\mu(k, l, m)-\mu \cdot \mu(k, l) \\
& -\mu \cdot(\mu(l-k, m-k)-\mu \cdot \mu(l-k)+\mu(l, m)-\mu \cdot \mu(l)+\mu(k, m)-\mu \cdot \mu(k)) \\
& -\left(\mu(k)-\mu^{2}\right) \cdot\left(\mu(m-l)-\mu^{2}\right)-\left(\mu(l)-\mu^{2}\right) \cdot\left(\mu(m-k)-\mu^{2}\right) \\
& -\left(\mu(l-k)-\mu^{2}\right) \cdot\left(\mu(m)-\mu^{2}\right)+\mu^{2} \cdot\left(\mu(m)+\mu(m-k)+\mu(m-l)-3 \mu^{2}\right) \\
= & \alpha^{m-l} \cdot\left(\alpha^{2 l+k} \cdot f_{4} \cdot \alpha^{2}(1-\alpha)\left(2+2 \alpha-\alpha^{2}\right)-(\beta+2) \cdot f_{3} \cdot \alpha^{2 l+1}\right. \\
& \left.+\frac{f_{2} \cdot \alpha^{2 l-k}}{(1-\alpha)\left(1-\alpha^{2}\right)} \cdot(\alpha+2 \beta)-\alpha^{2(l-k)} \cdot f_{3} \cdot \alpha \beta+\frac{\beta+1}{1-\alpha} \cdot \mu(k, l)-f_{2} \cdot \mu(k) \cdot(\alpha+\beta(1+\alpha))\right) \\
& -f_{1} \cdot\left(\frac{\beta+1}{1-\alpha} \cdot f_{2} \cdot \alpha^{m-k}+f_{1} f_{2} \cdot \alpha^{m-l}-f_{3} \cdot(1-\alpha) \cdot \alpha^{m+l-2 k+1}+\frac{\beta+1}{1-\alpha} \cdot f_{2} \cdot \alpha^{m}\right. \\
& \left.-f_{3} \cdot(1-\alpha) \cdot \alpha^{m+l+1}+f_{1} f_{2} \cdot \alpha^{m-l}+\frac{\beta+1}{1-\alpha} \cdot f_{2} \cdot \alpha^{m}+f_{1} f_{2} \cdot \alpha^{m-k}\right) \\
& \left.-f_{3} \cdot(1-\alpha) \cdot \alpha^{m+k+1}\right)-f_{2}^{2} \cdot\left(\alpha^{m-l+k}+2 \alpha^{m+l-k}\right)+f_{1}^{2} f_{2} \cdot\left(\alpha^{m}+\alpha^{m-k}+\alpha^{m-l}\right) \\
= & \alpha^{m+l+k+2} \cdot f_{4} \cdot(1-\alpha)\left(2+2 \alpha-\alpha^{2}\right)-(\beta+2) \cdot f_{3} \cdot \alpha^{m+l+1}+\frac{f_{2} \cdot \alpha^{m+l-k}}{(1-\alpha)\left(1-\alpha^{2}\right)} \cdot(\alpha+2 \beta) \\
& -\alpha^{m+l-2 k+1} \cdot f_{3} \cdot \beta+\frac{\beta+1}{1-\alpha} \cdot \alpha^{m-l} \cdot\left(\mu(k, l)-f_{1} \cdot \mu(k)\right)+\alpha^{m-l} \cdot f_{2} \cdot\left(\mu(k)-f_{1}^{2}\right) \\
& -f_{1} \cdot\left(\frac{2 \beta+1}{1-\alpha} \cdot f_{2} \cdot \alpha^{m-k}+2 f_{1} f_{2} \cdot \alpha^{m-l}-f_{3} \cdot(1-\alpha) \cdot \alpha^{m+l-2 k+1}\right. \\
& \left.+2 \frac{\beta+1}{1-\alpha} \cdot f_{2} \cdot \alpha^{m}-f_{3} \cdot(1-\alpha) \cdot \alpha^{m+l+1}-f_{3} \cdot(1-\alpha) \cdot \alpha^{m+k+1}\right) \\
& -f_{2}^{2} \cdot\left(\alpha^{m-l+k}+2 \alpha^{m+l-k}\right)+f_{1}^{2} f_{2} \cdot\left(\alpha^{m}+\alpha^{m-k}+2 \alpha^{m-l}\right) \\
= & \alpha^{m+l+k+2} \cdot f_{4} \cdot(1-\alpha)\left(2+2 \alpha-\alpha^{2}\right)-2 \cdot f_{3} \cdot \alpha^{m+l+1}+\frac{f_{2} \cdot \alpha^{m+l-k+1}}{(1-\alpha)\left(1-\alpha^{2}\right)} \\
& +\frac{\beta+1}{1-\alpha} \cdot \alpha^{m-l} \cdot\left(\frac{\beta+1}{1-\alpha} \cdot f_{2} \cdot \alpha^{l}+f_{1} f_{2} \cdot \alpha^{l-k}-f_{3} \cdot(1-\alpha) \cdot \alpha^{l+k+1}\right)+f_{2}^{2} \cdot \alpha^{m-l+k} \\
& -f_{1} \cdot\left(\frac{\beta+1}{1-\alpha} \cdot f_{2} \cdot \alpha^{m-k}+\frac{\beta+2}{1-\alpha} \cdot f_{2} \cdot \alpha^{m}-f_{3} \cdot(1-\alpha) \cdot \alpha^{m+k+1}\right)-f_{2}^{2} \cdot \alpha^{m-l+k} \\
= & \alpha^{m+l+k+2} \cdot f_{4} \cdot(1-\alpha)\left(2+2 \alpha-\alpha^{2}\right)-2 \cdot f_{3} \cdot \alpha^{m+l+1}+\frac{f_{2} \cdot \alpha^{m+l-k+1}}{(1-\alpha)\left(1-\alpha^{2}\right)} \\
& +\frac{f_{2}}{(1-\alpha)^{2}} \cdot \alpha^{m}-f_{3} \cdot \alpha^{m+k+1} \\
= & \alpha^{m} \cdot f_{4} \cdot\left((1-\alpha)\left(2+2 \alpha-\alpha^{2}\right) \cdot \alpha^{l+k+2}-\left(1-\alpha^{4}\right) \cdot\left(2 \cdot \alpha^{l+1}+\alpha^{k+1}\right)\right. \\
& \left.+\left(1+\alpha+\alpha^{2}\right)\left(1+\alpha^{2}\right) \cdot\left(1+\alpha+\alpha^{l-k+1}\right)\right) .
\end{aligned}
$$

For the corresponding central moment, we have

$$
\begin{aligned}
& \tilde{\mu}(k, l, m)=E\left[\left(X_{t}-\mu\right)\left(X_{t+k}-\mu\right)\left(X_{t+l}-\mu\right)\left(X_{t+m}-\mu\right)\right] \\
& =E\left[\left(X_{t} X_{t+k} X_{t+l}-\mu \cdot X_{t} X_{t+l}-\mu \cdot X_{t+k} X_{t+l}+\mu^{2} \cdot X_{t+l}\right) \cdot X_{t+m}\right] \\
& \quad-E\left[\left(\mu \cdot X_{t} X_{t+k}-\mu^{2} \cdot X_{t}-\mu^{2} \cdot X_{t+k}+\mu^{3}\right) \cdot X_{t+m}\right]-\mu \cdot \tilde{\mu}(k, l) \\
& =\mu(k, l, m)-\mu \cdot(\mu(l-k, m-k)+\mu(l, m)+\mu(k, l)+\mu(k, m)) \\
& \quad+\mu^{2} \cdot(\mu(k)+\mu(l)+\mu(m)+\mu(l-k)+\mu(m-k)+\mu(m-l))-3 \mu^{4} \\
& =\kappa(k, l, m)+\left(\mu(k)-\mu^{2}\right) \cdot\left(\mu(m-l)-\mu^{2}\right)+\left(\mu(l)-\mu^{2}\right) \cdot\left(\mu(m-k)-\mu^{2}\right) \\
& \quad+\left(\mu(m)-\mu^{2}\right) \cdot\left(\mu(l-k)-\mu^{2}\right) \\
& =\kappa(k, l, m)+f_{2}^{2} \cdot \alpha^{m-l+k}+2 \cdot f_{2}^{2} \cdot \alpha^{m+l-k} .
\end{aligned}
$$

Appendix A.3. Proof of Formula (4)

A Poisson random variable $Y \sim P o(\lambda)$ has the moment generating function $(\mathrm{mgf}) \mu_{Y}(z)=$ $\exp \left(\lambda\left(e^{z}-1\right)\right)$. Using that $X_{t}$, conditioned on $X_{t-1}, \ldots$, is Poisson distributed according to 
$\operatorname{Po}\left(\beta+\alpha \cdot X_{t-1}\right)$, see Definition 1.1, it follows that the mgf of $J_{t}$ is given by

$$
\begin{aligned}
\mu_{J}(z) & =E\left[\exp \left(z\left(X_{t}-X_{t-1}\right)\right)\right]=E\left[\exp \left(-z X_{t-1}\right) \cdot E\left[\exp \left(z X_{t}\right) \mid X_{t-1}, \ldots\right]\right] \\
& =E\left[\exp \left(-z X_{t-1}\right) \cdot \exp \left(\left(\beta+\alpha \cdot X_{t-1}\right)\left(e^{z}-1\right)\right)\right] \\
& =\exp \left(\beta\left(e^{z}-1\right)\right) \cdot E\left[\exp \left(-z X_{t-1}\right) \cdot \exp \left(\alpha\left(e^{z}-1\right) \cdot X_{t-1}\right)\right] \\
& =\exp \left(\beta\left(e^{z}-1\right)\right) \cdot \mu_{X}\left(\alpha\left(e^{z}-1\right)-z\right) .
\end{aligned}
$$

Taking the logarithm on both sides, we obtain the relation of formula (4).

\section{Appendix A.4. Proof of Proposition 3.1}

Since $J_{t}=\left(X_{t}-\mu\right)-\left(X_{t-1}-\mu\right)$, we can express moments of $J_{t}$ in terms of the central moments given in Corollary 2.2. For this purpose, we need the following results:

$$
\begin{aligned}
& \tilde{\mu}(0,1)=f_{3} \cdot \alpha\left(1+2 \alpha^{2}\right), \quad \tilde{\mu}(1,1)=f_{3} \cdot \alpha\left(1+\alpha+\alpha^{3}\right), \\
& \tilde{\mu}(0,0,0)=f_{4} \cdot\left(1+6 \alpha^{2}+5 \alpha^{3}+6 \alpha^{5}\right)+3 f_{2}^{2}, \quad \tilde{\mu}(0,0,1)=\alpha \cdot \tilde{\mu}(0,0,0), \\
& \tilde{\mu}(0,1,1)=f_{4} \cdot \alpha\left(1+\alpha+2 \alpha^{2}+6 \alpha^{3}+4 \alpha^{4}+4 \alpha^{6}\right)+f_{2}^{2} \cdot\left(1+2 \alpha^{2}\right), \\
& \tilde{\mu}(1,1,1)=f_{4} \cdot \alpha\left(1+3 \alpha+\alpha^{2}+5 \alpha^{3}+5 \alpha^{4}+2 \alpha^{5}+\alpha^{7}\right)+3 f_{2}^{2} \cdot \alpha .
\end{aligned}
$$

Now it follows that

$$
\begin{aligned}
& E\left[J_{t}^{2}\right]=E\left[\left(X_{t}-\mu\right)^{2}+\left(X_{t-1}-\mu\right)^{2}-2\left(X_{t-1}-\mu\right)\left(X_{t}-\mu\right)\right]=2(\tilde{\mu}(0)-\tilde{\mu}(1))=2 f_{2} \cdot(1-\alpha) ; \\
& E\left[J_{t}^{3}\right]=\tilde{\mu}(0,0)-3 \tilde{\mu}(1,1)+3 \tilde{\mu}(0,1)-\tilde{\mu}(0,0) \\
& \quad=3 f_{3} \cdot \alpha\left(\left(1+2 \alpha^{2}\right)-\left(1+\alpha+\alpha^{3}\right)\right)=-3 f_{3} \cdot \alpha^{2}(1-\alpha)^{2} ; \\
& E\left[J_{t}^{4}\right]=2 \tilde{\mu}(0,0,0)-4 \tilde{\mu}(1,1,1)+6 \tilde{\mu}(0,1,1)-4 \tilde{\mu}(0,0,1) \\
& \quad=2(1-2 \alpha) \cdot \tilde{\mu}(0,0,0)-4 \tilde{\mu}(1,1,1)+6 \tilde{\mu}(0,1,1) \\
& \quad=2(1-2 \alpha) \cdot f_{4} \cdot\left(1+6 \alpha^{2}+5 \alpha^{3}+6 \alpha^{5}\right)-4 f_{4} \cdot \alpha\left(1+3 \alpha+\alpha^{2}+5 \alpha^{3}+5 \alpha^{4}+2 \alpha^{5}+\alpha^{7}\right) \\
& \quad+6 f_{4} \cdot \alpha\left(1+\alpha+2 \alpha^{2}+6 \alpha^{3}+4 \alpha^{4}+4 \alpha^{6}\right)+2(1-2 \alpha) \cdot 3 f_{2}^{2}-12 f_{2}^{2} \cdot \alpha+6 f_{2}^{2} \cdot\left(1+2 \alpha^{2}\right) \\
& =2 f_{4} \cdot\left(1-2 \alpha+6 \alpha^{2}-7 \alpha^{3}-10 \alpha^{4}+6 \alpha^{5}-12 \alpha^{6}\right)-4 f_{4} \cdot\left(\alpha+3 \alpha^{2}+\alpha^{3}+5 \alpha^{4}+5 \alpha^{5}+2 \alpha^{6}+\alpha^{8}\right) \\
& \quad+6 f_{4} \cdot\left(\alpha+\alpha^{2}+2 \alpha^{3}+6 \alpha^{4}+4 \alpha^{5}+4 \alpha^{7}\right)+6 f_{2}^{2} \cdot\left(1-2 \alpha-2 \alpha+1+2 \alpha^{2}\right) \\
& =2 f_{4} \cdot\left(1-\alpha+3 \alpha^{2}-3 \alpha^{3}-2 \alpha^{4}+8 \alpha^{5}-16 \alpha^{6}+12 \alpha^{7}-2 \alpha^{8}\right)+12 f_{2}^{2} \cdot(1-\alpha)^{2} \\
& =2 f_{4} \cdot(1-\alpha)^{2}\left(1+\alpha+4 \alpha^{2}+4 \alpha^{3}+2 \alpha^{4}+8 \alpha^{5}-2 \alpha^{6}\right)+12 f_{2}^{2} \cdot(1-\alpha)^{2} .
\end{aligned}
$$

